\title{
La formation de l'objet du travail médical
}

L'exemple de la médecine générale et de la gynécologie obstétrique

The shaping of the medical work object: the example of general medicine and obstetrics

\section{Anne-Chantal Hardy}

\section{OpenEdition}

\section{Journals}

Édition électronique

URL : https://journals.openedition.org/travailemploi/6545

DOI : $10.4000 /$ travailemploi.6545

ISSN : $1775-416 \mathrm{X}$

Éditeur

DARES - Ministère du Travail

Édition imprimée

Date de publication : 1 janvier 2015

Pagination : 9-24

ISSN : 0224-4365

Référence électronique

Anne-Chantal Hardy, «La formation de l'objet du travail médical », Travail et Emploi [En ligne], 141 |

janvier-mars 2015, mis en ligne le 01 janvier 2017, consulté le 04 juillet 2022. URL : http://

journals.openedition.org/travailemploi/6545; DOI : https://doi.org/10.4000/travailemploi.6545 


\title{
La formation de l'objet du travail médical : l'exemple de la médecine générale et de la gynécologie obstétrique
}

\author{
Anne-Chantal Hardy(*)
}

L'objet du travail peut être défini par ce qui donne du sens à une activité, compris à la fois comme sa direction et sa signification. Est-il possible de caractériser un objet commun du travail médical pour l'ensemble de la profession, dans un contexte où tous les médecins sont spécialistes et exercent des métiers très différents? Après une interrogation sur l'objet du travail et les différentes façons de l'interroger, l'auteure tente de se saisir de la partie commune de cet objet, celle qui s'enseigne durant les six premières années d'études. Dans un second temps, l'étude de deux spécialités médicales, la médecine générale et la gynécologie obstétrique, montre deux formes d'évolutions différentes des objets du travail médical, fortement articulées à leur formation. Cela permet une problématisation de l'articulation entre formation pratique et formation théorique qui pourrait s'appliquer à l'ensemble des métiers de la santé, voire au-delà.

On a tous, quelque part dans nos souvenirs, au moins une scène en tête : qu'il s'agisse de Charcot présentant une hystérique à ses confrères ${ }^{(1)}$, d'images d'opérations chirurgicales ou de dissections de cadavres effectuées au beau milieu d'un amphithéâtre, la formation médicale se représente à travers une pratique montrée et regardée. Hospitalisés à notre tour et faisant l'expérience de la troupe d'étudiants pénétrant notre chambre derrière «le professeur», nous nous disons que c'est peutêtre cela qu' «apprendre au lit du malade» signifie.

Comment comprendre précisément cette expression : «au lit du malade»? Les médecins se forment auprès des lits d'hôpitaux, mais se forment-ils vraiment à partir du malade lui-même ? Est-ce le malade qui fournit la matière de l'enseignement ou est-elle faite d'autre chose ? Qu'apprend-on à voir aux étudiants, en leur «montrant» des malades ?

Pour Eliot FreIDSON (1970, p. 172), observant l'exercice de la médecine dans des cabinets américains de l'après-guerre, il semble évident que le travail du médecin est avant tout un travail pratique : «Il est par nature peu théorique, c'est plutôt un travail d'application et c'est en quoi il diffère nettement de celui du chercheur.» Cette constatation mérite deux commentaires.

Tout d'abord, le sociologue américain observe une pratique essentiellement consultante, à une

(*) Droit et changement social (UMR CNRS 6297), université de Nantes; anne-chantal.hardy@univ-nantes.fr

(1) Nous pensons par exemple ici à la célèbre peinture d'André Brouillet représentant le professeur Charcot enseignant l'hystérie à l'hôpital de la Salpêtrière, à la fin du XIX siècle. époque où la médecine restait encore très largement clinicienne. La pensée clinique est en effet d'abord pragmatique, comme l'explique Michel Foucault (1963, p. XIV), à travers «le changement infime et décisif qui a substitué à la question : "Qu'avezvous ?", par quoi s'inaugurait au XVIII e siècle le dialogue du médecin et du malade $[\ldots]$, cette autre où nous reconnaissons le jeu de la clinique et le principe de tout son discours : "Où avez-vous mal ?"». La question laisse entendre que c'est à partir de la souffrance exprimée par le malade que s'élabore la maladie. Ce qui se voit est un praticien qui répond à une demande s'exprimant le plus souvent en termes de souffrances; il tente alors de «résoudre les problèmes concrets des individus» (FREIDSON, op. cit.). Or, FouCAULT avait bien compris qu'il s'agissait au contraire d'une certaine façon de produire un discours autonome sur la maladie, révélée par le signifiant «symptôme». L'empirisme clinicien pouvait donc faire illusion dans les années cinquante, mais l'avènement de la médecine anticipatrice, permettant de faire advenir la maladie en dehors de toute expérience symptomatique, oblige à réinterroger la lecture de FREIDSON.

Cela nous conduit ensuite à la nécessité d'opérer une distinction entre le travail et l'objet du travail. Le travail est ce qui se passe concrètement et l'objet du travail, ce qui donne le sens de l'activité. Ainsi, nous pouvons poser l'hypothèse que si le travail du médecin (en particulier clinicien) consiste à répondre aux demandes de ses patients, pour autant, ce qui guide son action n'est pas de même nature. Le problème dont se saisit le médecin est sans doute différent de celui du patient. 
Pour mieux approcher cette distinction, le concept d'objet du travail oblige le sociologue à interroger concrètement le travail médical à partir de ce qui lui donne du sens, en tentant de rompre avec de fortes prénotions dans ce domaine. En effet, nous avons tous une idée de ce qu'est le travail médical, ne serait-ce que pour y avoir été soumis à un moment ou à un autre. La question mérite alors d'être traitée avec des outils conceptuels et méthodologiques susceptibles de permettre au sociologue, toujours potentiellement patient, de prendre la distance nécessaire à ses observations et surtout, à son interprétation.

Bien qu'universitaire, la formation des médecins est très largement composée de stages pratiques. S'il est difficile d'évaluer la proportion exacte d'enseignement théorique et d'enseignement pratique étant donné la diversité des organisations selon les facultés et selon les spécialités, on peut néanmoins considérer que ces enseignements représentent des volumes à peu près équivalents. Faire des hôpitaux des lieux de formation et de soins afin d'assurer un véritable apprentissage pratique aux futurs médecins fut même au cour de la réforme des études médicales portée par Robert Debré en 1958 (JAMOUS, 1969).

L'idée selon laquelle la formation des médecins est d'abord pratique est renforcée par les praticiens eux-mêmes. Ces derniers considèrent souvent qu'ils n'apprennent vraiment leur métier que lors du troisième cycle, au moment de leur internat, alors qu'ils n'ont que très peu d'enseignement théorique et qu'ils sont déjà considérés comme des professionnels. L'étudiant n'apprendrait donc surtout qu'à être étudiant, comme le suggéraient Howard BECKER et ses collègues (2005 [ 1 re éd. 1961]), le métier s'apprenant en le pratiquant. Et comme le médecin semble se former au contact des malades, nous pourrions penser que l'objet de sa formation - et donc de son travail - est, tout simplement, le malade. Plus encore, ce malade peut alors représenter un objet du travail commun à l'ensemble des professionnels de santé(2). C'est cette apparente évidence que nous nous proposons d'interroger dans ce texte en posant la question : comment se forme l'objet du travail médical?

Pour répondre à cette interrogation, nous nous appuierons sur quatre ensembles de données combinées et synthétisées. Le premier ensemble

(2) Certains pourraient penser que la question se situe entre l'objet et le sujet et c'est d'ailleurs contre la négation du sujet-malade que certains médecins tentent de défendre une médecine plus subjective (DuBAs, 2004). Notre propos ne concerne pas cette opposition, car il s'agit de considérer l'objet, non par rapport à la personne, mais par rapport au travail. Le concept d'objet, ici, n'est pas une notion concrète, mais une notion pratique : affirmer qu'il n'y a pas de travail sans objet ne consiste donc pas à dire que le sujet est objectivé, au sens de ramené au stade d'objet, mais que le travail porte sur (et est porté par) un (ou plusieurs) objet(s) qui lui donne(nt) du sens. est constitué des nombreux travaux portant sur les professions, les groupes professionnels et les évolutions du concept de profession, au-delà de la médecine. Le deuxième est issu d'enquêtes de terrain que nous avons réalisées à différents moments depuis le début des années 1990, essentiellement au sein de la profession médicale ${ }^{(3)}$, mais pas seulement. Le troisième s'est construit au fil du temps, de façon plus informelle, en maintenant une veille sur l'évolution de la formation médicale, en participant à cette formation en lien avec des départements de médecine générale, en assistant à des congrès d'internes et en exploitant des documents universitaires et professionnels. Le quatrième ensemble de données, enfin, a été recueilli à l'occasion de séances d'observation du travail des professionnels de santé en bloc obstétrical ${ }^{(4)}$. Ces observations s'intègrent dans un corpus de travaux en cours portant sur les pratiques de santé périnatale et les formes de collaborations professionnelles produites dans ce champ, dont nous livrons ici quelques résultats partiels.

Dans un premier temps, nous nous arrêterons donc sur ce concept d'objet du travail et sur les différentes façons de l'interroger. Défini d'abord comme un processus, cet objet se laisse particulièrement bien voir à certains moments de son élaboration : lors des recrutements ou des apprentissages. Dans un deuxième temps, nous prendrons deux exemples de spécialités professionnelles, la médecine générale et la gynécologie obstétrique et nous verrons comment évoluent les objets du travail dans ces deux champs d'exercice de la médecine. Cela nous conduira enfin à problématiser l'articulation entre formation pratique et formation théorique dans le champ, plus large, des professions de santé.

\section{L'objet du travail médical saisi par sa formation}

\section{Partir de l'objet du travail}

La sociologie du travail a accordé, depuis Georges Friedmann (1950), une grande importance à l'observation des travailleurs en poste, en décrivant leurs activités, pour comprendre

(3) Les premiers travaux collectifs ont été livrés au début des années 2000 et portaient sur les effets de la réforme de l'internat de médecine de 1982; puis d'autres travaux se sont intéressés aux études médicales (GADÉA, HARDY-DUBERNET et al., 2005) et aux choix des étudiants à l'issue des épreuves classantes nationales (Hardy-Dubernet, Faure, 2006.). Plus récemment, une série d'observations directes a été réalisée en bloc obstétrical de centre hospitalier universitaire (CHU), en complément de travaux menés en collaboration avec l'école des sages-femmes de Nantes.

(4) Dans un bloc obstétrical, autrement appelé «salle de naissance», travaillent régulièrement des sages-femmes, des médecins (gynécologues obstétriciens et anesthésistes), des aides-soignantes et auxiliaires de puériculture, des infirmiers anesthésistes et, ponctuellement, des pédiatres, en plus des cadres du service, sages-femmes de profession. 
comment ils s'arrangent de leurs contraintes afin de se préserver quelque autonomie, de quelles façons ils construisent leur professionnalisme, sur quels accords ils mènent leurs collaborations et à partir de quoi ils formulent leurs revendications. En France, la sociologie des professions semble aujourd'hui revenir à ces observations du travail, en particulier depuis que la problématique de la professionnalisation est utilisée pour un ensemble de plus en plus large de métiers (Demazière, Gadéa, 2009; Boussard et al., 2010). En effet, la distinction entre, d'une part, des professions regardées comme «emblématiques et achevées» (Demazière, 2009, p. 84) au premier rang desquelles se trouvent les médecins, et, d'autre part, des "groupes professionnels» (Chapoulie, 1973) étudiés dans la tradition de l'école de Chicago, permet de relativiser la catégorisation souvent brutale et parfois un peu artificielle entre les «vraies» professions et les métiers, dont on est parfois réduit à constater la "professionnalisation » (Ріотет, 2002; Demazière, 2008). La sociologie des professions, et notamment la sociologie médicale, a plus souvent regardé les enjeux internes au groupe professionnel et ses recompositions que la pratique et l'exercice du métier(5). En fait, l'autonomie professionnelle est souvent considérée comme une caractéristique tellement évidente du travail médical, et l'expertise scientifique si difficile à décrypter pour un profane, que les études portant sur l'activité des médecins s'attachent peu à l'analyse du sens de l'acte lui-même. Cela nécessite d'ailleurs un travail complexe de retraduction des échanges entre patients et médecins afin de déconstruire la pensée experte, comme l'a tenté Aaron V. Cicourel (2002).

L'objet du travail peut être défini par le processus qui donne du sens à l'activité de travail (HARDY, 2013). Le sens ici est à la fois la direction et la signification. Cet objet n'est jamais prédéfini, que ce soit par les professionnels ${ }^{(6)}$, les institutions ou les usagers. L'objet du travail est surtout un concept opératoire et un outil méthodologique conduisant à interroger, à partir d'un angle particulier, une activité professionnelle en postulant au départ que l'on ignore de quoi il s'agit. Si cet exercice peut être spontané concernant des professions qui nous sont inconnues, il est plus difficile pour des activités dans lesquelles nous sommes tous impliqués et qui nous sont familières. Ainsi le travail des médecins

(5) À quelques exceptions près, comme le travail de Jean Peneff (1997) portant sur les chirurgiens ou celui de Nicolas DODIER (1993) sur les médecins du travail.

(6) S'il est nécessaire de bien comprendre comment le travail prend sens pour les professionnels eux-mêmes, comme par exemple l'a fait Alexandra BIDET (2010) au sujet du «vrai travail», le concept d'objet du travail va au-delà de l'autoévaluation des acteurs et inclut les processus de formation - initiale et continue - et de sélection des membres du groupe professionnel. nous semble accessible et bien connu puisque nous avons déjà eu recours à ces professionnels et avons eu l'occasion de les voir "en exercice». Pourtant, c'est dans le cas de professions proches que cette interrogation de l'objet du travail nous semble la plus heuristique. En effet, ce concept devient alors un outil de déconstruction des prénotions auxquelles le sociologue est toujours plus ou moins soumis. Comment en effet étudier «objectivement» un travail dont nous pensons avoir été, sommes, ou serons le sujet/l'objet ? Comment ne pas se placer quelque part dans cette relation entre le soignant et le soigné ? S'il n'est pas possible, ni même souhaitable de neutraliser le regard de l'observateur, il nous a semblé utile, à de nombreuses reprises, de s'armer d'outils permettant de guider l'interprétation de l'image qu'il capte.

D'un point de vue général, tout objet du travail peut être investigué à partir de questions assez simples, centrées sur ses dynamiques : comment se recrutent les professionnels ? Comment sontils évalués ? Qu'est-ce qui définit la qualité d'un travail ? À quel moment commence, se termine le travail ? En particulier, la formation est un moment privilégié pour étudier la mise en forme de cet objet, car pour être appris et incorporé par le professionnel, il doit être rendu visible, être dit, sous une forme ou sous une autre. Ce qui prend de la valeur dans une formation permet de mieux comprendre de quoi l'objet lui-même est constitué. Les formes de gratification, tant matérielles que symboliques, sont des indicateurs puissants de la nature d'un objet du travail.

\section{Quel est l'objet du travail médical ?}

L'éclatement des conditions d'exercice de la médecine en même temps que des spécialités conduirait à penser qu'il n'y a pas un objet du travail médical commun à l'ensemble de la profession. En fait, tout dépend de la position de l'observateur. Si celui-ci étudie les médecins au travail, il peut en effet considérer qu'il n'y a quasiment pas de point commun entre le médecin biologiste et le médecin généraliste, entre le chirurgien et le médecin scolaire, etc. Pourtant, tous ont reçu une formation commune d'au moins six années (voir encadré 1), qui ne peut pas être ignorée : c'est bien là qu'un premier objet se forme pour tous les médecins. L'objet du travail commun aux médecins se trouve donc d'abord dans ces six premières années : c'est là que nous sommes allée le chercher. 


\section{Encadré 1 \\ La formation des médecins}

Les médecins sont formés dans les universités, au sein de facultés dont le fonctionnement est particulier. D'abord, l'inscription en première année est ouverte à tous les bacheliers, même si la quasi-totalité des étudiants de deuxième année sont des bacheliers scientifiques (LABARTHE, HerAULt, 2003). Ensuite, l'accès à la deuxième année se fait, depuis 1972, à l'issue d'un concours dont le nombre de places, fixé depuis 1983 par le ministère de la Santé, a connu des fluctuations importantes : établi à plus de 12000 la première année du concours, il est tombé à 3500 au début des années 1990 pour remonter aujourd'hui à plus de 7000 . Depuis 2011, la première année est commune aux filières de médecine, dentaire, pharmacie et maïeutique (sages-femmes) et, dans certaines facultés, de kinésithérapie.

Le système licence-master-doctorat (LMD), sur lequel les facultés de médecine doivent s'aligner aujourd'hui, est assez mal calé sur le déroulement des études médicales.

Celles-ci sont en effet organisées traditionnellement selon trois cycles. Le premier, de deux ans, correspond à l'apprentissage des matières fondamentales; le second, d'une durée de quatre ans, à celui de la sémiologie et de la thérapeutique. Ces six premières années sont validées par les universités mais se concluent par des épreuves classantes nationales (ECN) qui visent à affecter à tous les étudiants en médecine de France, un rang selon lequel chacun choisira sa filière et sa région d'affectation pour son troisième cycle. Ces épreuves remplacent, depuis 2004, le concours de l'internat de spécialité. Durant les trois dernières années du deuxième cycle, les étudiants sont considérés comme «étudiants hospitaliers " car ils sont en stage la moitié de leur temps et touchent une rémunération hospitalière. Ce statut succède à l'ancien statut «d'externe », terme encore usité pour le désigner.

La thèse de médecine, enfin, donne lieu au titre de doctorat d'exercice mais n'est pas assimilée au grade de doctorat. Dans le système LMD, ce diplôme est donc équivalent à un master. Pour accéder aux postes d'enseignants à l'université, les étudiants doivent ainsi entreprendre parallèlement un master et un doctorat de sciences, le plus souvent en sciences de la vie, plus rarement en sciences humaines. Les facultés de médecine proposent depuis peu des filières de doctorat.

Il n'est pas possible ici d'entrer dans le détail de ce qui caractérise cet apprentissage commun, du moins dans ses effets sur la formation d'un objet médical partagé. Cependant, nous pouvons synthétiser quelques-unes de ses principales dimensions à partir de couples conceptuels qui entrent parfois en opposition sans pour autant strictement se contredire, et constituent les supports dialectiques d'une pensée commune parfois paradoxale : formation/sélection; théorie/pratique; général/spécialisé. Chacun d'entre eux fait apparaître des systèmes de combinaison, d'articulation ou d'opposition permettant, in fine, de mieux appréhender la formation de l'objet commun du travail médical.

\section{La combinaison formation / sélection}

Les médecins constituent une profession réglementée et, jusqu'à présent, extrêmement stable, dans la mesure où les diplômés de médecine qui exercent une autre profession sont aujourd'hui assez rares. Ainsi, la relation formation emploi en médecine est probablement l'une des plus fortes qui existent, y compris parmi les professions de santé(7). De ce fait, tout admis en deuxième année de médecine a de fortes probabilités d'exercer ce métier toute sa vie. Cette proximité exceptionnelle entre le titre et le poste (Bourdieu, Boltanski, 1975) conduit les pouvoirs publics à réaliser des prévisions démographiques dont les conséquences, dans un système de soins reposant en grande partie sur le secteur libéral, sont déterminantes. Le concours de fin de première année, aujourd'hui commun à quatre professions médicales, vaut donc quasiment concours de recrutement et impacte fortement la structure du groupe professionnel. En fin de sixième année, une autre épreuve répartit les futurs médecins dans les filières de spécialité. Ces épreuves classantes nationales (ECN), qui ne sont ni un concours (il y a plus de postes que de candidats) ni un examen (les notes obtenues ne sont pas validantes), visent exclusivement à classer chaque année les milliers d'étudiants en médecine et à les répartir en internat de spécialité dans les centres hospitalo-universitaires français. Il s'agit donc d'une épreuve distribuant les individus dans les différents postes d'internes ouverts sur le territoire. Autrement dit, cette procédure s'apparente à un vaste recrutement national des différentes spécialités médicales, puisant dans le stock des étudiants en médecine inscrits en sixième année dans les universités françaises. L'affectation se fait au choix, selon les postes restant à disposition pour chaque individu en fonction de son rang (HARDY-DuberNet, FAURE, 2006).

Ces deux épreuves sont uniquement des épreuves théoriques, organisées en grande partie sur la base de connaissances évaluées de façon systématique, puisque leur fonction est avant tout classante. Ce n'est qu'après les ECN que les étudiants sont débarrassés de cette obligation de classement et peuvent réellement se consacrer à l'apprentissage du métier qu'ils ont, de

(7) Il est cependant difficile de mesurer cette stabilité et son évolution. En effet, les principales enquêtes d'insertion excluent les filières universitaires de santé de leur champ, comme si la question était résolue a priori (CALMAND, 2013). Dans le même temps, les données fournies par le Conseil de l'ordre des médecins montrent que, d'une part, les jeunes médecins mettent plus de temps que leurs aînés à s'installer, mais aussi, d'autre part, qu'un nombre croissant de médecins quittent le tableau de l'Ordre avant leur retraite. Ce dernier point semble indiquer qu'ils se dirigent vers d'autres professions, mais les travaux de la Direction de la recherche, des études, de l'évaluation et des statistiques (Drees) ne confirment pas, pour l'instant, cette fuite (BILLAUT, 2006). 
gré ou de force, choisi. Si les étudiants réalisent des stages hospitaliers assez tôt dans leurs études, ceux-ci sont l'objet de validation mais pas de notation et n'interviennent pas dans les systèmes de classement. De ce fait, il existe une confusion entre l'objectif de formation, qui exprime la nécessité, pour tous, d'acquérir un socle commun de connaissances, et l'objectif de sélection, qui vise à discriminer les étudiants entre eux. Ainsi, la première partie de la formation médicale, représentée par les six premières années, est centrée sur l'apprentissage de notions théoriques, où la réalité du malade est secondaire. C'est à partir de matières dites «fondamentales» que s'apprennent les maladies, lesquelles ne sont qu'ensuite incarnées par des malades «en vrai». Cette forme d'apprentissage oblige les médecins à réorganiser leurs connaissances théoriques pour que celles-ci deviennent des aides au diagnostic et à la décision thérapeutique le moment venu. La domination de la logique classante sur la logique formatrice s'inscrit dans un système hiérarchisant la compétitivité des candidats, dont les résultats se traduisent en une plus ou moins grande liberté de choix du poste d'interne, donc de la spécialité. Autrement dit, plus le candidat est «performant», plus l'étendue de ses possibles sera vaste; moins il se lancera dans cette compétition, moins il aura de choix pour son futur métier. La forte contrainte des étudiants est à la mesure de la grande liberté revendiquée par les professionnels. Cette logique, qui ressemble à celle d'une bureaucratie méritocratique, apparaît aussi fondamentalement «libérale» dans le sens où la mise en concurrence des étudiants est censée élever les performances de l'ensemble du groupe et permettre des régulations «naturelles» de l'offre de soins. Il s'agit là d'un système aujourd'hui largement remis en cause mais qui a sous-tendu longtemps les réformes des études médicales.

\section{L'articulation théorie I pratique}

Sur le fond, les six premières années des études médicales sont également très structurantes pour un collectif ensuite éclaté en différentes spécialités. Ce qui rassemble cette communauté professionnelle est l'apprentissage théorique, valeur centrale de l'évaluation, tandis que les premiers terrains de stage remplissent une fonction plus informatrice (ils apprennent à l'étudiant à se situer dans l'univers hospitalier) que formatrice (les stagiaires sont assez passifs $\left.{ }^{(8)}\right)$. L'organisation pédagogique au fil des années témoigne de cet objet élaboré au plus fort de son abstraction, à travers des matières différenciées (la chimie, la biologie, les statistiques, etc.) avant de se centrer sur l'étude des signes des maladies (la sémiologie) et leur traitement. La «maladie du

(8) Nos travaux ont mis en évidence différentes phases d'activité des stagiaires selon les années. Qualifiés de «thrombus de couloir» durant les premières années, tant ils encombrent les services sans rien faire, ils apprennent souvent par eux-mêmes à se rendre utiles. Cet apprentissage est aussi celui de l'autonomie. médecin» se forme à partir de ses phénomènes biologiques avant même ses manifestations corporelles; elle préexiste au malade et à son corps et peut s'étudier sans que ce dernier en exprime quoi que ce soit. Cette rupture, d'une part, avec les représentations que chaque étudiant pouvait avoir du champ de la médecine avant le début de ses études, et, d'autre part, avec les non-professionnels de santé devenus alors les «profanes», est au cœur du mécanisme de structuration de la profession par l'élaboration progressive d'un objet commun. Il s'agit donc, de ce point de vue, d'une épreuve pratique, puisqu'elle déplace concrètement les individus d'un monde à un autre.

À aucun moment du cursus, théorie et pratique ne font «parts égales» car on commence par la théorie et on termine par la pratique. Cette articulation est structurante et produit une hiérarchie dans la valeur des apprentissages. Si les médecins disent qu'ils apprennent leur métier lors de leur internat et «sur le terrain », c'est parce qu'ils se confrontent aux autres et s'inscrivent alors dans un vrai rapport social et professionnel. Le passage de la personne souffrante au «patient» s'opère en même temps que le passage du «profane» au médecin, mais cette prise de position ne peut avoir lieu qu'après la construction de l'objet théorique.

L'apprentissage théorique fait l'objet d'une évaluation commune à l'ensemble des étudiants et organisée de façon centralisée par les ministères de tutelle et les universités. Il fait donc lien entre tous. En revanche, la pratique et ensuite l'attribution des postes hospitaliers et universitaires sont largement laissés à la subjectivité des pairs et à la cooptation (9). S'il existe aujourd'hui quelques faibles contrôles de la "qualité pédagogique» des services, aucune évaluation pratique n'est réalisée en présence d'un enseignant extérieur ${ }^{(10)}$. Ici encore, l'apprentissage de la valeur du professionnel va de pair avec l'acquisition d'une certaine notion de sa liberté, essentiellement pratique.

Dès son premier jour d'internat, le futur médecin est devenu quasiment un professionnel jouissant du pouvoir de prescrire, acte symbolique de la position médicale. Paradoxalement, les internes les mieux classés, dits les plus «brillants », sont souvent ceux qui, jusque-là, ont privilégié l'apprentissage théorique à l'apprentissage pratique si bien que leur prise de fonction est parfois problématique. La théorie peut alors devenir leur refuge et la recherche

(9) Par exemple, la possibilité de faire carrière à l'hôpital est laissée à l'appréciation d'un chef de service qui désigne ceux qui pourront prétendre à un poste dans son service. De même, les postes de maîtres de conférences et de professeurs ne sont pas ouverts à la concurrence; le plus souvent, il n'y a qu'un seul candidat, déjà désigné et pour qui le poste est mis au «concours». (10) Ce qui est le cas dans les écoles de sages-femmes ou d'infirmières : le stage est évalué et validé par les référents du stagiaire dans le service, mais les enseignants se déplacent aussi sur les terrains de stage pour évaluer en pratique les étudiants. 
leur salut, tant le «malade» devenu réel, avec ses symptômes flous, ses plaintes démesurées ou ses proches trop présents, vient perturber l'étude et le traitement de la «maladie » ${ }^{(11)}$.

Ce n'est, en tout cas, pas à partir du malade que l'objet du travail s'est élaboré et ce n'est pas sur sa capacité à «entendre» celui-ci que le jeune médecin peut justifier sa pratique. Au début de l'internat, la maladie n'est pas encore incorporée, elle est un sujet d'examen théorique, un ensemble de connaissances abstraites.

C'est bien dans l'articulation entre théorie et pratique que l'on perçoit donc la spécificité de cette formation qui, bien que laissant une place importante aux «stages», s'organise sur une référence avant tout abstraite - ce qui permet de s'interroger sur la nature de l'apprentissage en situation de travail des professionnels de santé, en fonction de la place qu'occupent les différentes phases d'enseignement dans le curriculum général de la formation.

\section{L'opposition générall spécialisé}

Traditionnellement et jusqu'au début des années 1980, la médecine était "générale». On peut même dire, comme le décrivait Michel ArLiaud (1987, p. 92) dans les années 1980, qu'il n'y avait «pas, pour l'essentiel, de discontinuité institutionnellement établie dans l'espace professionnel». Même si des hiérarchies professionnelles se manifestaient en interne, chacun pouvait se référer à une «médecine officielle, primordialement générale et libérale» (loc. cit.). Jusqu'en 1983, tous les médecins étaient, d'abord, des médecins généralistes, ce métier étant celui qui était appris durant les sept premières années des études médicales, la spécialisation intervenant dans un second temps. La plupart des médecins, y compris les internes, effectuaient au cours de leurs études des remplacements de médecine générale, s'essayant par l'expérience à une pratique. Certains y trouvaient de l'intérêt tandis que d'autres préféraient se spécialiser, le plus souvent en constatant que cette médecine-là était beaucoup plus compliquée que celle qui ne s'occupe que d'une fonction ou d'un organe.

La notion de spécialisation médicale est une notion particulièrement complexe, qui trouve son origine dans plusieurs logiques et se concrétise par des mécanismes parfois contradictoires. Il y a donc, selon le point de vue choisi, différentes façons de l'aborder.

L'une d'elles est de penser la spécialisation comme le résultat d'une segmentation du corps professionnel, à la suite des travaux des sociologues interactionnistes, en particulier d'Everett C. HugHeS

(11) Certains jeunes médecins que nous avons rencontrés défendent ainsi l'idée d'une médecine «sans malade» qui serait beaucoup plus performante, car débarrassée des scories de l'interaction humaine, dès lors que l'ensemble des données (résultats biologiques, radiologiques, etc.) peuvent être accessibles par dossier informatisé.
(1996) et d'Anselm L. Strauss (1961; 1992). En étudiant le travail hospitalier, les auteurs constatent une division du travail conduisant à la création de segments dont les contours sont sans cesse redéfinis. Le processus de professionnalisation est alors considéré comme des "agrégations de segments poursuivant des objectifs divers, plus ou moins subtilement maintenus sous une appellation commune à une période particulière de l'histoire» (STRAUSS, $1961 ; 1992$, p. 69). Ce processus prend cependant des formes diverses selon les pays et la spécialisation ne s'opère pas de la même façon en France, en Grande-Bretagne ou en Allemagne (WeIsz, 2006).

La spécialisation peut aussi être pensée comme le résultat d'ajustements et de négociations interinstitutionnelles. En France, ce mouvement est dû à la conjonction de plusieurs évolutions, liées en particulier au système de formation et à la volonté des pouvoirs publics, via la Sécurité sociale, de légiférer sur l'exercice des spécialités (PINelL, 2004). Si la concordance entre les filières de troisième cycle, les diplômes de spécialité et les spécialités reconnues par la Sécurité sociale est aujourd'hui quasi totale, ce ne fut pas toujours le cas. Pendant longtemps, des systèmes parallèles et parfois discordants de reconnaissance de la spécialité ont coexisté : le Conseil national de l'ordre des médecins pouvait reconnaître à un praticien la qualification de spécialiste sans qu'il ait obtenu de diplôme dans le domaine; les universités pouvaient délivrer les certificats d'études spécialisées sans que celles-ci ouvrent le droit de bénéficier de la tarification de l'acte de spécialiste établie par la Sécurité sociale. Devant la complexité à définir ce qui relevait de la médecine spécialisée, il semble que le problème ait été résolu dès lors que la médecine générale est devenue une spécialité médicale. La médecine n'a d'unité que le nom et une formation commune, mais au niveau de la pratique, elle est fortement segmentée.

Enfin, cette logique de spécialisation ne correspond pas à un mode de division unique du travail médical. En effet, certaines spécialités sont populationnelles (les femmes, les enfants, les personnes âgées, les travailleurs, etc.), d'autres concernent un organe (la peau, les yeux, etc.), un appareil (l'appareil digestif, l'appareil cardiovasculaire, l'appareil locomoteur, etc.) ou une fonction (l'endocrinologie, l'hématologie, etc.); d'autres spécialités relèvent de techniques particulières, thérapeutiques (la chirurgie) ou diagnostiques (la radiologie, la biologie); enfin, certaines sont consacrées à des ensembles de maladies (les maladies infectieuses, rhumatismales, cancéreuses, mentales, etc.). Aujourd'hui, ces spécialités s'entrecroisent : les chirurgiens sont spécialisés dans une partie du corps ou un type de chirurgie; les pédiatres se spécialisent dans certaines maladies infantiles, démultipliant ainsi les champs d'exercice de la médecine.

Ces nombreux métiers s'exercent en outre aujourd'hui dans des conditions très variées, les 
médecins pratiquant souvent des exercices multiples, combinant une (ou des) activité(s) salariée(s) avec un exercice libéral, alternant entre le secteur public et le secteur privé. La médecine d'abord «générale et libérale» d'il y a une trentaine d'années semble ainsi avoir disparu. Pourtant et malgré cette diversité, il y a bien un objet commun à tous ces métiers, que nous avons défini par celui de la guérison (HARDY, 2013), entendu ici d'abord comme un processus qui guide l'activité médicale dans ses diverses dimensions. Cet objet du travail commun se décline de façons différentes selon le mécanisme de spécialisation qui l'a transformé, et cela peut, en particulier, s'observer à travers la formation pratique des spécialistes.

\section{La formation d'objets spéciaux}

La deuxième partie de la formation initiale des médecins s'effectue «en cours d'emploi», à savoir sur des postes d'internes. Elle diffère selon les spécialités : elle dure entre trois (médecine générale) et cinq ans (spécialités chirurgicales) et peut être prolongée dans le cadre d'un diplôme d'études spéciales complémentaires (DESC) et d'un clinicat. La pratique médicale, sur le terrain, est une pratique de spécialité apprise avant tout à l'hôpital. Le lieu d'exercice de l'interne peut donc être nettement différent du lieu de la pratique professionnelle, si celle-ci se déroule ensuite en cabinet libéral ou en clinique privée. Derrière cette homogénéisation apparente des procédures de formation, de fortes variations subsistent, du fait même de l'écart entre apprentissage et pratique, entre objet théorique et actes concrets, entre exercice de la spécialité et contours de la discipline. Il est ainsi difficile de dresser un paysage homogène du processus de formation d'un objet à multiples facettes. C'est pourquoi nous prenons ici le parti d'approcher ce processus selon une perspective comparative et dynamique. Nous aborderons la formation de deux spécialités à partir de ce qui fonde leur principale référence distinctive. Ainsi, la médecine générale s'est constituée en spécialité non sur la base de sa pratique, mais sur celle de sa formation, instituant un paradoxe à l'origine d'une profonde difficulté de ce segment professionnel à devenir une discipline universitaire. L'obstétrique, discipline ancienne mais dont les contours sont poreux, s'est calée sur la médicalisation de l'événement de la naissance, en s'appuyant sur le principe du risque pour se construire un objet original.

\section{La médecine générale : une spécialisation par la formation}

\section{Une spécialisation de forme}

Le mouvement de spécialisation de la médecine ayant abouti à la suppression de la médecine générale comme référence de la profession pour ne plus admettre que des «spécialités médicales», s'observe à travers les évolutions du système de formation et des modes d'apprentissage du métier (pour en savoir plus sur les effectifs de médecins généralistes, voir encadré 2). L'imposition de la spécialisation s'est en effet concrétisée, dans le système de formation, selon trois voies : le système de sélection, les contenus théoriques de formation et l'organisation des stages pratiques. La formation du médecin référée à la spécialité n'est pas la même que la formation du médecin d'abord générale; le modèle change.

La médecine générale a en fait été la dernière spécialité à faire l'objet d'une sélection par le rang. Avant 1983, il existait plusieurs modèles de choix d'un exercice médical, chacun relevant d'un système de valeurs différent. Les internes des hôpitaux étaient considérés comme l'élite, choisie de deux façons : d'abord parce qu'ils réussissaient des concours réputés très difficiles, mais aussi parce qu'une fois en poste, c'était les chefs de service qui désignaient ceux qu'ils allaient orienter dans leur spécialité. Le concours de l'internat était un concours d'accès aux postes hospitaliers mais non un concours de spécialité, celle-ci étant choisie ensuite par les internes. Ceux qui ne voulaient pas faire l'internat mais souhaitaient néanmoins se spécialiser avaient toujours la possibilité de passer un certificat d'études spéciales (CES), mais celuici ne donnait accès qu'aux spécialités médicales. C'est cette hiérarchie des sélections qui a donné sa réputation de spécialité d'excellence à la chirurgie. En effet, les spécialités chirurgicales n'étaient accessibles que par l'internat, les chirurgiens étant attachés à la qualité d'une formation pratique volontiers présentée comme un «compagnonnage» (Bercot, Matthieu-Fritz, 2007; Zolesio, 2012 ; article de Régine Bercot dans ce numéro). Traditionnellement, s'opposaient donc deux modèles de formation qui d'ailleurs allaient puiser leurs références dans le conflit ancien entre chirurgiens et médecins: l'un, intellectuel, nourri de l'acquisition de savoirs abstraits; l'autre, pratique, enseigné sur le terrain et dans les hôpitaux ${ }^{(12)}$.

En 1983, toutes les spécialités furent rendues accessibles par l'internat, médecine générale exclue. Ce changement eut deux conséquences : l'accès aux spécialisations fut désormais subordonné au rang de classement des candidats, mais tous étaient formés

(12) Les évolutions de la science médicale ne sont pas sans effet sur les évolutions des prestiges des professions. Si les chirurgiens ont gagné leurs lettres de noblesse, c'est en grande partie parce que les anesthésiques et les antibiotiques leur ont permis d'opérer plus facilement et de limiter les effets iatrogènes de leurs actes. De ce fait, les chirurgiens ont obtenu des résultats de plus en plus spectaculaires, jusqu'à ce que la médecine découvre de nouvelles méthodes thérapeutiques et de nouvelles classes de médicaments. Ainsi, la cardiologie, spécialité médicale, est devenue une spécialité «reine» dans les choix des étudiants, car elle condense tous les atouts d'un exercice recherché : un corpus de connaissances limité et facilement maîtrisable, des résultats particulièrement spectaculaires, des actes à la fois techniques et cliniques, une rentabilité tout à fait confortable et une symbolique très valorisée étant donné l'organe principal concerné. 


\section{Encadré 2 \\ Les médecins généralistes}

Compter les médecins généralistes est une opération délicate car il faut faire une distinction entre les diplômés de médecine générale et les praticiens de la médecine générale (BLOY, SCHWEYER, 2010). En effet, on estime à environ $40 \%$ des diplômés de médecine générale, les médecins exerçant une activité autre que celle du «médecin de famille» (ONDPS, 2008).

Au 1er janvier 2013, on comptait 101803 médecins qualifiés en médecine générale, pour une population médicale totale en exercice de 218296 praticiens (SICARD, 2013a). Leur densité est très variable sur le territoire. À peine les deux tiers de ces médecins exercent en cabinet (62\%), les autres travaillant à l'hôpital public (16\%) ou dans divers établissements privés et associatifs. Surtout, la pyramide des âges montre une réduction très forte des effectifs pour les plus jeunes générations.

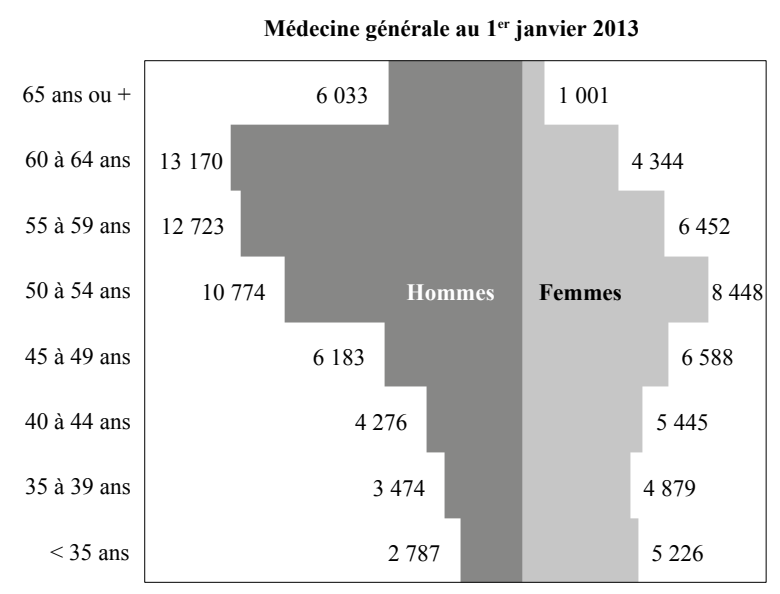

La médecine générale est très féminisée aujourd'hui, mais cela n'était traditionnellement pas le cas. Ainsi en 2013, 62\% des généralistes âgés de moins de 40 ans étaient des femmes alors qu'elles n'étaient que $27 \%$ parmi les généralistes âgés de 55 ans et plus.

Les revenus des médecins généralistes sont parmi les plus bas des médecins libéraux, d'autant plus que seulement $11 \%$ d'entre eux sont rattachés au secteur 2, c'està-dire bénéficiant d'une autorisation de dépassement d'honoraires par rapport au tarif conventionnel (BELLAMY, MıKOL, 2012). Cette possibilité est quasiment fermée aux jeunes générations : il faut en effet désormais avoir été chef de clinique pour bénéficier de ces tarifs et les postes sont très rares en médecine générale.

* Agence des systèmes d'information partagés de santé.

** Répertoire partagé des professionnels de santé.

par l'internat. Entre 1983 et 2004, la médecine générale connut un statut particulier : pas encore reconnue comme spécialité, mais plus vraiment commune à tous. N'étant pas intégrée à l'internat, elle devint la filière obligée de ceux échouant ou ne passant pas l'internat, et fut rapidement associée à une filière de relégation. Pourtant, les promoteurs de cette réforme de 1983 espéraient ainsi «revaloriser» la filière générale en lui accordant un réel troisième cycle (le résidanat). Mais la force du processus sélectif prédomina et, plus encore qu'auparavant, ce furent les étudiants les moins "performants» qui intégrèrent cette discipline ${ }^{(13)}$.

À partir de 2004, intronisée comme «spécialité», la médecine générale devient une filière choisie parmi toutes les autres à l'issue du classement des ECN. Depuis, elle regagne progressivement du terrain sur les autres spécialités. En effet, de moins en moins d'étudiants s'y orientent par défaut, même si les derniers classés n'ont souvent pas d'autres possibilités que d'opter pour la médecine générale (GODEFroy, 2013).

Parallèlement, les contenus théoriques des deux premiers cycles ont été modifiés. En effet, jusqu'en 2004, il existait un programme de formation

(13) Cette évaluation de la «performance» est ici purement académique : il s'agit en fait des étudiants qui avaient les moins bonnes notes aux examens universitaires. universitaire et un programme de préparation au concours d'internat. Le premier était dispensé par les universités en vue de former l'ensemble des étudiants à une médecine commune, tandis que le second était centré sur des savoirs spécialisés afin de sélectionner les futurs internes. Mais progressivement, pour améliorer leurs scores à l'internat, les facultés ont calé leurs programmes et leur mode d'enseignement sur celui du concours, aboutissant en 2004 à un seul et même programme, de fait orienté vers les savoirs spécialisés. Ainsi, l'apprentissage théorique lui-même s'est transformé, privilégiant des connaissances sectorisées autour de pathologies spécialisées au détriment des connaissances transversales auparavant dispensées par les universités. Durant ces périodes intermédiaires, de forts écarts dans les enseignements s'observaient entre les facultés, selon qu'elles se donnaient comme objectif de former en priorité des spécialistes ou des généralistes.

Du point de vue de la formation pratique, les choses se sont compliquées. En effet, s'il s'agit d'offrir aux étudiants un enseignement de terrain, quel terrain est le plus adapté à la médecine générale ? Concrètement, pour former des praticiens à une médecine libérale en cabinet, c'est auprès des praticiens de ville que les internes doivent apprendre. Pour autant, le principe même de l'exercice libéral est peu adapté à l'accueil de stagiaires. Il a donc fallu mettre en place un système mixte en 
recrutant des praticiens maîtres de stage susceptibles d'accueillir des internes, mais sans garantir une formation homogène ni même une qualité pédagogique facilement contrôlable (BLOY, 2005). Le décalage entre la formation hospitalière et la formation en cabinet, dans un contexte de dévalorisation de l'omnipratique de ville par des médecins hospitaliers ayant pour modèle l'expertise spécialisée, a contribué à un malaise chez les apprentis généralistes, les conduisant à redouter la pratique libérale et à se tourner vers d'autres formes d'exercice de leur métier.

C'est donc dans sa forme que la médecine générale s'est spécialisée, plus que dans son objet, puisque cette discipline ne correspond pas vraiment à une innovation pratique et technique bouleversant la division du travail médical. Ainsi, c'est a posteriori que les étudiants et les enseignants procèdent à une redéfinition de leur objet de sorte qu'il puisse se distinguer, cette fois sur la base de la pratique, des autres objets spécialisés.

\section{Une spécialité anomique?}

Comment alors se forme l'objet du travail de la médecine générale ? L'institutionnalisation d'une formation sur le modèle de la médecine spécialisée a contribué à brouiller les pistes. En effet, la médecine générale est devenue une spécialité parce qu'elle a été soumise aux mêmes procédures de sélection et de formation que les spécialités médicales, mais pas à l'issue d'une redéfinition de son objet. Paradoxalement, elle s'en est trouvée à la fois réduite, puisqu'elle ne concernait plus la totalité des médecins, et renforcée : dans le nouveau paysage composé exclusivement de spécialistes, elle est devenue la spécialité numériquement la plus importante. Dans la même période, la pratique elle-même n'a pas été repensée et l'exercice du métier, s'il a évolué, n'a pas été restructuré fondamentalement par cette spécialisation. La principale difficulté que rencontre aujourd'hui la médecine générale «spécialisée» est d'élaborer un objet qui puisse être reconnu de la même façon que les autres spécialités.

Devant ces difficultés, la recherche en médecine générale s'attelle plus à définir cet objet qu'à en alimenter le contenu. En effet, ce que l'on voit apparaître est un modèle d'approche, dit biopsychosocial (ENGEL, 1980), où les références à d'autres disciplines que celles des sciences médicales traditionnelles sont mobilisées. Il s'agit de proposer à la fois une «approche globale» du patient et d'acquérir une légitimité reposant sur le modèle de l'Evidence Based Medicine (EBM) ${ }^{(14)}$. Les autres spécialités ont, quant à elles, émergé à l'occasion d'un processus de différenciation de la médecine générale, fondé sur une population, une pratique ou un organe. La médecine générale est prise au piège de son propre mouvement de «valorisation» et ne peut pas se différencier sur ce mode. Le parti pris est donc de se distinguer de l'ensemble des autres spécialités par une approche originale, se rapportant davantage à un processus, une "manière de faire» qu'à un objet lui-même. Dans un système de formation organisé sur la base de savoirs spécialisés, l'approche dite "globale» de la médecine générale correspond à une autre socialisation professionnelle. Comme certains le font remarquer, ils doivent « apprendre à l'envers », puisqu'il s'agit de partir du patient pour trouver la maladie. Ainsi, durant le troisième cycle des études médicales, une attention plus importante est portée, par les enseignants universitaires, à l'arbre décisionnaire, à l'écoute des signes, à l'environnement du patient, aux inégalités sociales, etc. - autant d'éléments qui ne figurent plus dans l'enseignement général de la médecine, ou sous des formes standardisées (comme les sciences humaines et sociales [SHS] dont le statut reste très flou, ou l'éthique, parfois relayée au rang de discipline optionnelle).

Cette situation paradoxale place la médecine générale dans une situation proche de l'anomie. Nous utilisons cette notion dans une perspective durkheimienne (Durkheim, 1981 [1930, $1^{\text {re éd.]) }}$ dans la mesure où ce n'est pas l'absence d'inscription collective qui est en jeu ici, mais bien la cohérence des valeurs de référence permettant au groupe de se retrouver autour d'un objet partagé. $\mathrm{Au}$ mieux, les médecins généralistes pourraient se retrouver autour d'un mandat sociétal représenté par le rôle d'orientation confié au «médecin traitant». Mais la discipline médicale est ici absente de la définition; pour des étudiants dont l'ensemble de la formation s'est organisée autour d'un système de valeurs reposant sur la performance intellectuelle, cette identification peut être problématique.

(14) L'Evidence Based Medicine, traduit le plus souvent en français par médecine basée sur les preuves, tend à modifier le paradigme de la réflexion clinique reposant auparavant surtout sur l'expérience du clinicien, par un paradigme décisionnel fondé sur les preuves apportées par la science, et accessibles par la littérature scientifique et les métaanalyses. L'EBM s'inscrit dans un ensemble plus vaste d'usages de la statistique comme outil de preuve, tels qu'ils se sont développés au $\mathrm{Xx}^{\mathrm{e}}$ siècle autant en médecine que dans les politiques publiques ou les arbitrages économiques (DesRosières, 2008). 


\section{L'obstétrique : la formation d'un objet risqué}

\section{Encadré 3 \\ Les gynécologues obstétriciens}

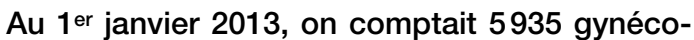
logues obstétriciens, se répartissant à peu près à parts égales entre l'hôpital public (2377) et le secteur privé, en cabinet libéral (2289) et établissements privés. Il s'agit d'une des populations médicales qui a vu ses effectifs le moins chuter. II faut toutefois mettre en rapport ce constat avec la quasi-disparition de la filière de gynécologie médicale depuis les années 1980.

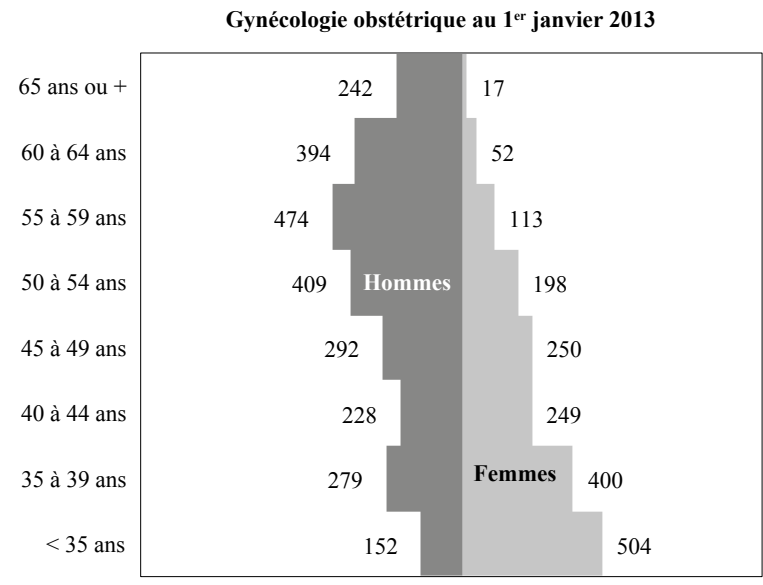

Source: Asip-Santé RPPS, traitements Drees (SICARD, 2013a).

La profession compte en moyenne 41,9\% de femmes, avec une forte féminisation dans les tranches d'âges les plus jeunes. Ce basculement est d'autant plus remarquable qu'il s'agit d'une filière chirurgicale, discipline encore assez peu féminisée (cf. les articles de Régine Bercot et Emmanuelle Zolesio dans ce numéro de la revue).

Le revenu des gynécologues obstétriciens est relativement élevé. Mais il est difficile d'en avoir une réelle mesure, car une partie d'entre eux, les obstétriciens, est en général comptabilisée avec les chirurgiens. Notons en outre que les gynécologues obstétriciens peuvent connaître des carrières différentes, car bon nombre d'entre eux abandonnent l'obstétrique à un moment donné pour se consacrer essentiellement à la gynécologie, chirurgicale ou seulement médicale.

L'obstétrique est une spécialité qui n'est pas organisée autour d'une maladie, mais plutôt d'un événement: la naissance (pour en savoir plus sur les effectifs de gynécologues obstétriciens, voir encadré 3). Celui-ci n'est pas un événement médical, même s'il est médicalisé, car il existe en dehors de la médecine (ce qui n'est pas le cas de la cancérologie qui nécessite une approche particulière de la maladie) et d'un système de santé (qui crée le médecin généraliste). Sur quelle référence intervient donc le médecin au cours de la naissance et en quoi cet objet est-il spécifique par rapport aux sages-femmes, par exemple?

La réponse théorique et "officielle» est celle de la distinction entre l'accouchement «normal» (eutocique) et l'accouchement pathologique (JACQUES, 2007). Autrement dit, ce qui fait la différence entre les deux est précisément l'intervention du médecin, les sages-femmes assurant en fait des accouchements ne nécessitant pas d'acte réservé aux médecins à un moment donné (par exemple l'usage de forceps). Les frontières entre ces différents métiers sont donc assez instables. C'est finalement la notion de risque, elle-même assez peu définie, qui contribue à les dessiner.

\section{Une filière aux frontières mouvantes}

La gynécologie obstétrique est une filière appartenant aux disciplines chirurgicales. Mais elle présente la particularité de se situer à la frontière de plusieurs champs de pratiques ${ }^{(15)}$. D'abord, elle comprend à la fois la gynécologie et l'obstétrique. Il s'agit d'une spécialité centrée sur une population (les femmes), des organes (l'appareil génital féminin) et une période (la maternité). En outre, elle est à la fois médicale et chirurgicale, et s'intéresse à des phénomènes physiologiques non pathologiques (comme la contraception ou la naissance). Cette spécificité la place en concurrence avec d'autres spécialités médicales, comme la médecine générale ou l'endocrinologie (qui est l'étude des phénomènes hormonaux) mais aussi d'autres professions, en particulier les sages-femmes (profession dite médicale, mais séparée du corps médical auquel elle est subordonnée par ses institutions).

La France est l'un des rares pays européens à avoir développé, dans l'après-guerre, une spécialité médicale de gynécologie, séparée de la chirurgie et de l'obstétrique (16). Cette filière de formation a été supprimée en 1983 au profit de la filière unique de gynécologie obstétrique, intégrée alors aux filières chirurgicales. Si aujourd'hui la gynécologie médicale a été rouverte par l'intermédiaire de

(15) En dehors des spécialités non prescriptives (comme la médecine du travail) ou de laboratoire, les spécialités sont divisées en spécialités médicales et chirurgicales. En fait, la chirurgie a d'abord constitué une spécialité (la chirurgie générale) à elle seule, s'opposant à la médecine. Puis le mouvement de spécialisation a entraîné une division au sein de ces deux modes d'exercice, voire parfois un couplage des spécialités. Par exemple, la dermatologie et l'otorhinolaryngologie (ORL) sont à la fois médicales et chirurgicales, et leurs cursus suivent les maquettes de formation en chirurgie. La gynécologie-obstétrique fait partie de ces spécialités mixtes et ceux qui la choisissent peuvent donc aussi n'exercer que la gynécologie médicale, ce qui est le cas de certains médecins en fin de carrière, désireux d'éviter les gardes par exemple.

(16) Une spécificité due en particulier au lobbying féministe défendant les traitements hormonaux face aux traitements chirurgicaux des pathologies gynécologiques (WeIsZ, LöwY, 2005). 
quelques postes à l'internat, elle reste très marginale et ne suffit pas à renouveler la population de ces spécialistes ${ }^{(17)}$. Concrètement, le suivi gynécologique des femmes peut aujourd'hui être assuré par des médecins généralistes, dont d'ailleurs un certain nombre acquiert des qualifications dans ce domaine, mais aussi, depuis peu, par les sagesfemmes ${ }^{(18)}$. Ces mêmes professionnels peuvent également assurer le suivi des grossesses dites non pathologiques. L'accouchement est pris en charge, dans les établissements publics et dans une partie des établissements privés, par les sages-femmes, en dehors de situations, là encore, pathologiques. Seuls les actes techniques et les opérations chirurgicales (gynécologiques et césariennes) sont pratiqués uniquement par les gynécologues obstétriciens.

Alors que l'obstétrique semble être une discipline clairement définie par la prise en charge d'un moment particulier, elle se trouve en fait quasiment délimitée par une notion difficile à stabiliser: la pathologie. Loin d'envisager le passage du «normal» au «pathologique» comme un continuum dont les frontières sont socialement autant que scientifiquement sans cesse redessinées, comme Georges CANGUILHEM (1966) incite à le penser, l'organisation des filières de spécialité impose une représentation marquée par la rupture, signifiée par le changement des intervenants et un basculement dans les règles de la prise en charge. Historiquement, la gynécologie obstétrique s'est d'ailleurs instituée en milieu hospitalier dans un contexte de lutte entre des hommes (médecins) et des femmes (sages-femmes et matrones ${ }^{(19)}$ ), non seulement pour le monopole d'une pratique, mais aussi celui d'un savoir. L'encouragement à l'accouchement en milieu hospitalier durant le $\mathrm{XIX}^{\mathrm{e}}$ siècle ne peut pas réellement être justifié par une meilleure sécurité, les femmes mourant alors fréquemment de fièvres puerpérales liées à des infections que l'on qualifie aujourd'hui de nosocomiales. Ces hospitalisations, au XIX ${ }^{\mathrm{e}}$ siècle, s'expliquent souvent par la nécessité, pour les médecins désireux de parfaire leurs techniques,

(17) En effet, l'âge moyen des gynécologues médicaux est de 59 ans et sur une population totale de 3600 spécialistes, il n'y a que 129 gynécologues de moins de 45 ans en exercice (SICARD, 2013a, p. 98 et suiv.).

(18) Celles-ci sont actuellement plus de 20000 en France, dont un cinquième environ exerce en secteur libéral (SICARD, 2013b). Elles sont considérées, mais de façon ambiguë, comme une «profession médicale» parce qu'elles ont un droit de décision et de prescription, bien que limité aux situations dites "physiologiques».

(19) Les matrones étant des femmes, souvent elles-mêmes mères de familles nombreuses, qui aidaient les femmes à accoucher en l'absence de professionnels de santé. Sagesfemmes et matrones se sont fait concurrence tout au long du $\mathrm{XIX}^{\mathrm{e}}$ siècle, pour les accouchements à domicile; les sagesfemmes ont d'ailleurs témoigné d'une belle capacité à se défendre, depuis leurs origines et même en milieu hospitalier (FAURE, 2005). de pratiquer des accouchements indépendamment des sages-femmes - ce qui leur permettait de se constituer un savoir autonome (ScHLUMBOHM, 2002).

\section{Guérir un risque}

En décrivant les activités du docteur Osiander, exerçant à la maternité de l'université de Göttingen au tout début du Xix ${ }^{\mathrm{e}}$ siècle, Jürgen $\mathrm{S}_{\text {chlumbohm }}$ (2002) montre que le praticien n'hésite alors pas à avoir recours à des extractions instrumentales lors de naissances qui ne le nécessitent pas, au seul motif d'entraîner ses étudiants ou à des fins de démonstration technique. Dès l'origine, la science obstétricale semble ainsi se définir avant tout comme une technique permettant aux médecins de maîtriser le cours de l'accouchement et d'instituer leurs propres normes. Cette spécialité apparaît comme une discipline autonome, rattachée de fait à la chirurgie donc à l'intervention directe, et non comme une branche de la maïeutique, qui définit les sciences et techniques de la naissance. Cette distinction est importante : elle clive fondamentalement les obstétriciens et les sages-femmes et il n'est pas anodin que le conflit refasse régulièrement surface.

L'observation du travail en salle de naissance(20) permet d'apporter une compréhension supplémentaire à cette question à partir de la façon dont se forme l'objet du travail pour chaque catégorie de personnels, en particulier les sages-femmes et les gynécologues obstétriciens.

En dehors des taxinomies médicales, nous avons repéré trois types d'accouchements, selon la manière dont se répartissent les fonctions des médecins et des sages-femmes. Le premier peut être défini comme «spontané» ou «naturel» même si, dans un grand centre hospitalier, il n'y a pas de naissance sans intervention technique et professionnelle. On peut cependant mettre dans cette catégorie les accouchements qui en

(20) La «salle de naissance» est le service où se déroulent les accouchements. Cette appellation est celle qu'affectionnent les sages-femmes, tandis que les médecins l'appellent «bloc obstétrical», signifiant une différence qui n'est pas anodine. L'affichage à l'hôpital est d'ailleurs celui des médecins. Ce «bloc» est par ailleurs adossé au «bloc gynécologique» où ont lieu les opérations de chirurgie gynécologique. Mais il présente aussi des similitudes avec un service d'urgence, parce que l'activité n'y est pas planifiée et dépend des arrivées des parturientes d'une part, et parce que le personnel organise sa présence, vingt-quatre heures sur vingt-quatre et sept jours sur sept, sur le mode des gardes, d'autre part. Nous avons réalisé des séries d'observations en salle de naissance d'un hôpital public, étalées sur une période de sept mois en 2012-2013. Nous avons suivi des personnels différents sur la totalité de leurs gardes en nous centrant particulièrement sur le travail des professionnels, leurs coopérations (ou absence de coopération), leurs limites et leurs complémentarités en tentant d'explorer une question centrale : peut-on définir un objet commun aux professionnels de santé travaillant en salle de naissance ou chacun s'empare-t-il d'un objet séparé ? 
requièrent le minimum et qui sont totalement gérés par les sages-femmes. Le deuxième type d'accouchement peut être qualifié de «dirigé», pour reprendre une expression appartenant au langage indigène. L'action des professionnels de santé consiste alors à "prendre en main » le déroulement du travail(21), c'est-à-dire à tenter d'en maîtriser les différentes phases, souvent pour en accélérer le mouvement, mais sans avoir recours à des techniques directement interventionnistes. En général, les sages-femmes prennent la décision de «diriger» un accouchement, mais elles préviennent les médecins et sont en contact avec eux afin qu'ils puissent intervenir en cas de besoin. Enfin, on distingue les accouchements que j'ai appelés «contrariés», dans la mesure où ce sont les médecins qui modifient le cours de l'accouchement, quelle que soit l'avancée du travail. Cette action peut, concrètement, prendre des formes très diverses : manipulation du foetus in utero, extraction instrumentale, jusqu'à la césarienne. Mais dans tous les cas, il se passe quelque chose qui vient «changer» le cours de l'accouchement, ou du moins qui cherche à le modifier (car l'action médicale est parfois compromise et le déroulement «spontané» finit par s'imposer).

De même que les médecins interviennent à un moment très particulier de certains accouchements et font basculer l'ensemble du processus, les internes sont, avant tout, formés à entrer en action à ce moment-là. On voit ici apparaître, en actes, une rupture réelle entre, d'une part, une représentation de l'accouchement en tant que processus conduisant à la naissance d'un enfant et, d'autre part, sa médicalisation, qui consiste à «prendre la main», c'est-à-dire à intervenir «contre» le processus naturel comme on va "contre» une maladie. Cette rupture est en outre signifiée par le terme utilisé pour désigner le patient du médecin : on ne parle pas d'«enfant» (des parents) mais de «fœetus». Celui-ci représente à la fois un tout et des parties (le «mobile fotal», la «tête fœtale», etc.), distinctes de son devenir d'enfant et désignées comme autant de problèmes médicaux décorporés (LEWIN, 2004) (22).

La formation des internes, qui présente quelques particularités, révèle de façon saisissante les ruptures conduisant à l'émergence de l'objet du travail obstétrical.

Elle consiste d'abord à apprendre à «faire des gestes » à partir de techniques précises. Par exemple, une interne m'explique qu'une des premières choses qu'elle a su faire au cours de son internat était des césariennes, alors même qu'elle n'avait

(21) Pour distinguer le travail de la parturiente de celui des professionnels, j'ai choisi de mettre le mot travail en italique quand il concerne celui des femmes en cours d'accouchement. (22) Par exemple, on parlera de «transfert de foetus in utero» pour désigner le changement de lieu d'hospitalisation d'une femme enceinte. jamais pratiqué d'accouchement physiologique. Les internes apprennent donc à «faire» avant d'apprendre à «prescrire» : ils s'entraînent à manipuler des forceps avant de savoir à quel moment les appliquer. Ils se forgent ainsi une représentation de leur geste indépendamment de ce qui s'est passé avant, comme si leur technique prenait le dessus sur l'ensemble du processus.

Dans un deuxième temps, ils apprennent le schéma d'une prise de décision : repérer les «risques», les évaluer et éventuellement agir en conséquence. Ils le font lors de staffs ${ }^{(23)}$ où des situations sont présentées et discutées avec les médecins seniors ou, plus formellement, à l'occasion d'enseignements reposant sur des études de cas. Ils apprennent aussi à consulter les "procédures» et les «bonnes pratiques» pour savoir ce à quoi ils «ont droit». Ils utilisent un vocabulaire volontiers légaliste : est-ce «interdit», a-t-on «le droit», est-ce «obligatoire», etc. ?

En dernier lieu, et quasiment au registre des apprentissages annexes, ils vont «voir» comment ça se passe «spontanément». Ils n'agissent pas ou peu dans ce cas, car dans les grands hôpitaux les étudiantes (24) sages-femmes ne leur laissent pas volontiers leur place. Cela peut les conduire à considérer l'accouchement physiologique comme un «non-événement», avec lequel ils doivent se familiariser comme on se socialise à un environnement professionnel.

$\mathrm{Au}$ final, le processus d'apprentissage du travail médical de l'accouchement diffère nettement de celui traditionnellement suivi par les sages-femmes. En effet, les étudiantes sagesfemmes pratiquent les gestes les plus techniques en fin de formation et apprennent d'abord la physiologie de l'accouchement et ses multiples manifestations. Le «normal» n'est pas homogène et c'est cette multiplicité des formes spontanées dont les sages-femmes deviennent spécialistes. En revanche, les médecins apprennent le geste maîtrisé : leur objet est ainsi de procéder à l'accouchement qu'ils projettent et de réussir la technique qu'ils mettent en œuvre quitte à contrarier l'évolution du travail.

Ce qui tient lieu de maladie dans ce cas de figure n'est pas la naissance ou l'accouchement, c'est « le risque». Cependant, le concept de risque doit ici être compris, non comme une hypothèse ou une probabilité, mais comme l'apparition réelle d'un désordre qui permet de déclencher l'action. Le

(23) Le staff est le terme utilisé dans les hôpitaux pour décrire les réunions d'équipes médicales amenées à prendre une décision. Il se distingue des réunions de service, qui peuvent rassembler l'ensemble des professionnels (médicaux et paramédicaux, voire de service) d'une unité de soins.

(24) Étant donné que plus de $95 \%$ des sages-femmes sont des femmes, nous accordons le féminin aux étudiantes. 
risque, c'est le mal. Cette conception très particulière est à l'origine de la phrase la plus répandue dans les salles de naissance et dans les cours d'obstétrique : "On sait qu'une naissance est sans risque quand tout s'est terminé sans problème." Lors de la grossesse par exemple, l'enjeu de la surveillance est l'élimination des risques avec, comme principe de base, l'idée qu'ils ne peuvent pas être tous éliminés, et en gardant donc à l'esprit que le danger est toujours présent. Le concept de «grossesse normale» a par conséquent disparu en France ${ }^{(25)}$, pour ne plus laisser exister que les grossesses à bas, moyen et haut risque, et un classement des maternités en fonction de leur capacité à gérer ces risques, évalués par les équipements de prise en charge des nouveau-nés.

L'étude de la formation de l'objet médical, du point de vue institutionnel comme pratique, permet de complexifier une représentation souvent trop homogène de la profession médicale. L'objet central et commun est bien celui de l'apprentissage d'un processus, la guérison, mais à partir d'une reconstruction préalable de l'objet «maladie» qui devient saisissable et même maîtrisable par le médecin et, d'une certaine façon, quitte le corps du malade. La guérison ici s'entend comme le processus de lutte contre la maladie, mais pas comme l'état du malade «rétabli». Le processus de guérison n'est possible que s'il s'applique à une maladie identifiée, quel que soit le ressenti du malade. C'est pourquoi il faut bien que cette maladie s'exprime, que ce soit à travers un risque pour permettre l'intervention médicale au cours de la grossesse, en dehors du déclenchement d'une pathologie et même, plus généralement, lorsqu'il s'agit de penser l'action préventive.

Ainsi, ce n'est pas l'existence d'une formation pratique qui fait du métier de médecin un métier concret. S'il l'est dans les faits et dans les gestes, il ne l'est pas nécessairement par son objet: ce constat permet d'éclairer un certain nombre de phénomènes observables de façon récurrente. Parmi eux, on peut citer les cris d'alarme concernant la médecine générale (BLOY, 2011), qui semble ne plus attirer les jeunes médecins, avec, pour conséquence, l'apparition de «déserts médicaux». Les médias se font régulièrement les porte-parole du désamour des étudiants pour ce métier et les professionnels, du malaise des praticiens. Les études semblent d'ailleurs montrer

(25) Car ce n'est pas le cas dans tous les pays occidentaux, comme le montrent les travaux de Madeleine AKRICH et Bernike PAsveer (1996) à propos de la naissance en France et aux Pays-Bas. des signes aggravés de burn out chez les généralistes, voire des suicides fréquents, associés à des conduites d'évitement à l'installation chez les plus jeunes, dans un contexte où ces derniers ne sont pas nécessairement moins sollicités que leurs aînés (26). S'il faut se méfier des analyses volontiers pessimistes produites par la profession médicale sur elle-même ${ }^{(27)}$, il faut aussi prendre en considération ces manifestations de désarroi, également relayées par les plus convaincus des professionnels, à savoir les enseignants.

Nous pourrions alors nous interroger sur le lien entre une perte d'objet de la moitié de la profession médicale - en effectifs - et ces signes de malaise. Cela montre que l'on ne peut pas instituer une discipline et, plus encore, une spécialité d'exercice, sans se soucier de la cohérence de l'objet qui la supporte et en constitue la référence. Ce sont des médecins généralistes qui ont exprimé l'idée qu'il leur fallait «réapprendre à l'envers » dès lors qu'ils se trouvaient en situation de partir des plaintes des patients. Comment alors concilier cette approche de la médecine consultante et celle de la biomédecine sur laquelle la recherche hospitalo-universitaire est organisée?

Les questions soulevées par la gynécologie obstétrique sont différentes mais complémentaires. Le travail de l'obstétricien ne s'apprend pas dans une continuité corrélée à l'événement de la naissance, mais à partir d'une logique de prise de pouvoir, à un moment donné, sur le travail des parturientes, en centrant son action sur «l'objet fœtal». On comprend alors la confrontation existant entre les sages-femmes et les obstétriciens, qui se manifeste à l'occasion de la demande des premières d'être reconnues comme une "profession médicale». Si l'objet médical s'est construit par opposition à la physiologie, reconnaître une qualification médicale à des professionnels qui prennent en charge cette physiologie reviendrait à dénaturer leur objet, voire à le vider de sa substance distinctive.

Enfin, ces résultats mettent l'accent sur la différence qui existe entre la formation «sur le terrain» et la formation «en cours d'emploi». Les anciennes générations de médecins étaient formées «en cours d'emploi», au détriment sans doute aussi de leurs patients. Ils optaient souvent pour une spécialisation après l'avoir un peu

(26) Sur l'extrême complexité et la très forte sollicitation des généralistes des générations précédentes, on peut lire le bel ouvrage de John BERGER et Jean MoHr (2009) qui retracent l'activité d'un médecin généraliste dans la campagne anglaise des années soixante.

(27) En effet, les travaux réalisés sur des panels de médecins généralistes mettent en évidence des situations de stress au travail très contrastées et pas nécessairement pires que pour l'ensemble des travailleurs, en particulier les autres catégories sociales supérieures (ORS, URML, 2010). 
pratiquée; ils s'exerçaient à la médecine générale avant d'avoir décidé d'y rester ou non (Herzlich et al., 1993) et pouvaient aussi changer d'orientation ou de pratique au cours de leur carrière. Aujourd'hui, les stages pratiques n'ont pas tout à fait la même fonction : ils s'inscrivent dans la suite d'un apprentissage théorique, sous la forme d'un processus translationnel (28).

Pour autant, il serait inexact de considérer que l'objet du travail médical est "formé» une fois pour toutes à l'issue des études de médecine. En effet, il est amené à se modifier tout au long d'une carrière et, le plus souvent, en dehors des structures hospitalo-universitaires. Plusieurs pistes, sans être exhaustives, méritent ici d'être soulevées, qui pourraient contribuer à comprendre comment, au-delà des cycles d'études, l'objet médical est mouvant et se transforme continuellement. La première relève des conditions d'exercice extra-hospitalières. Les deux spécialités étudiées dans ce texte sont ainsi soumises à d'autres aléas dans le secteur privé et libéral. Les médecins généralistes par exemple, sont confrontés, d'une part, à une patientèle dont les demandes varient dans le temps et, d'autre part, à des tutelles dont les exigences et les modes de contrôle sont de plus en plus fortes. Notamment, l'introduction d'une rémunération à la performance ou les nouvelles formes d'organisation des soins primaires contribuent à faire évoluer autant l'objet lui-même que le rapport à celui-ci. La définition d'objectifs collectifs ou de performances des soins est-elle susceptible de «déformer» l'objet du travail médical ?

Par ailleurs, si nous avons voulu distinguer, en milieu hospitalier, les positions formelles des sagesfemmes et des médecins face aux différents types d'accouchements, cette distinction ne vaut que dans un contexte assez particulier, qui est celui d'une structure hospitalo-universitaire. Dans les cliniques privées par exemple, y compris dans les cliniques de niveau $1^{(29)}$, où la plupart des accouchements présentent des risques réduits, les médecins interviennent au moment de la naissance, sans pour autant modifier le cours d'un travail eutocytique. Cette intervention est souvent économique dans la mesure où l'acte médical est rémunérateur. La tarification à l'activité de l'hôpital pose aussi ce type de problème. La "valeur» d'un acte n'est pas que symbolique ou médicale; elle est, dans notre système, également déterminée par un objectif de rentabilité.

Les évolutions scientifiques et les nouvelles façons de penser "la maladie» méritent également d'être prises en compte. En effet, la place de plus en plus importante de la médecine prédictive conduit les scientifiques à réclamer un autre mode de prise en charge des patients, y compris par les cliniciens. Si la maladie se pense à partir d'un patrimoine génétique ou de marqueurs biologiques, le dépistage plus ou moins automatique de ces prédispositions pourrait, à certains moments, dicter la prescription médicale. Par ailleurs, en attribuant à l'hôpital public une mission de technologie et de recherche "de pointe», on reporte sur le système ambulatoire et en majorité privé, la résolution des problèmes courants. Le travail médical ne risque-t-il pas d'être limité à l'application d'un objet forgé toujours plus loin du malade et de ce qu'il vit concrètement?

Enfin, nos résultats nous amènent aussi à interroger le modèle de la formation médicale au regard des autres formations de santé, en particulier l'articulation entre théorie et pratique dans un certain nombre d'entre elles. Le raccourcissement des études d'infirmières fut l'occasion d'une recomposition des périodes d'enseignement, privilégiant les savoirs théoriques en début d'études et les applications pratiques à la fin. Il en est de même pour les sages-femmes, dont les stages sont moins nombreux en début de formation. Si plusieurs filières médicales sont associées en première année de médecine, c'est pour les mettre en concurrence sur des disciplines abstraites. Si un jour les universités de santé rassemblent les principales professions médicales et paramédicales, le risque est que cette fusion s'opère sur le mode de la «spécialisation» de la médecine générale, c'està-dire en offrant le modèle des filières les plus valorisées pour redessiner la forme des métiers sans, pour autant, en repenser l'objet.
(28) La recherche translationnelle vise à étudier les modalités d'application des résultats des recherches réalisées en laboratoire, et testés au mieux sur des animaux. Il s'agit d'un volet important de la recherche biomédicale aujourd'hui, car souvent très problématique au regard des difficultés de coopération et des différences d'approche entre les chercheurs et les cliniciens. (29) Les maternités sont classées en trois catégories de niveau $(1,2$ et 3$)$ indiquant le niveau croissant d'équipement de réanimation néonatale. Les maternités de niveau 1 correspondent donc à celles où se pratiquent les accouchements dont le risque est estimé le plus bas. 


\section{Bibliographie}

Akrich M., Pasveer B. (1996), Comment la naissance vient aux femmes. Les techniques de l'accouchement en France et aux Pays-Bas, Le Plessis-Robinson, Synthélabo.

Arliaud M. (1987), Les médecins, Paris, La Découverte.

BASZANGER I. (1981), «Socialisation professionnelle et contrôle social : le cas des étudiants en médecine futurs généralistes », Revue française de sociologie, vol. 22, no 2, pp. 223-245.

Becker H. S., Geer B., Hughes E. C., Strauss A. L. (2005 [1961, 1 re éd.]), Boys in white. Student culture in medical school, New York, Transaction Publishers.

Bellamy V., Mikol F. (2012), «Les revenus d'activité des médecins libéraux: le poids des dépassements», Études et résultats, no 811 , Direction de la recherche, des études, de l'évaluation et des statistiques (Drees).

Bercot R., Mathieu-Fritz A. (2007), «La crise de recrutement des chirurgiens français: entre mythes et réalités », Revue française de sociologie, vol. 48, no 4, pp. 751-779.

Berger J., Mohr J. (2009), Un métier idéal : histoire d'un médecin de campagne, Paris, Éd. de l'Olivier.

Bidet A. (2010), «Qu'est-ce que le vrai boulot? Le cas d'un groupe de techniciens», Sociétés contemporaines, $\mathrm{n}^{\mathrm{o}} 78$, pp. 115-135.

Billaut A. (2006), «Les cessations d'activité des médecins », Études et résultats, no 484 , Drees.

Bloy G. (2005), «La transmission des savoirs professionnels en médecine générale : le cas du stage chez le praticien », Revue française des affaires sociales, no 1, pp. 101-125.

Bloy G. (2011), «Comment peut-on devenir médecin généraliste aujourd'hui ? Le renouvellement des médecins généralistes vu à travers une cohorte de jeunes diplômés », Revue française des affaires sociales, n 2-3, pp. 9-28.

Bloy G., Schweyer F.-X. (dir.) (2010), Singuliers généralistes. Sociologie de la médecine générale, Rennes, Presses de l'École des hautes études en santé publique.

Bourdieu P., Boltanski L. (1975), «Le titre et le poste : rapports entre le système de production et le système de reproduction", Actes de la recherche en sciences sociales, vol. 1, no 2, pp. 95-107.

Boussard V., Demazière D., Milburn P. (2010), L'injonction au professionnalisme. Analyses d'une dynamique plurielle, Rennes, Presses universitaires de Rennes.

Calmand J. (2013), «Les docteurs : une longue marche vers l'emploi stable», Bref du Céreq, no 316.
Canguilhem G. (1966), Le normal et le pathologique, Paris, Presses universitaires de France.

Chapoulie J.-M. (1973), « Sur l'analyse sociologique des groupes professionnels », Revue française de sociologie, vol. $14, \mathrm{n}^{\circ} 1$, pp. 86-114.

Cicourel A.-V. (2002), Le raisonnement médical. Une approche sociocognitive, textes réunis et présentés par P. Bourdieu et Y. Winkin, Paris, Seuil.

Demazière D. (2008), «L'ancien, l'établi, l'émergent et le nouveau: quelle dynamique des activités professionnelles?», Formation emploi, no 101, pp. 41-53.

Demazière D. (2009), «Posface. Professionnalisations problématiques et problématiques de la professionnalisation», Formation emploi, no 108, pp. 83-90.

Demazière D., Gadéa C. (dir.) (2009), Sociologie des groupes professionnels: acquis récents et nouveaux défis, Paris, La Découverte.

Desrosières A. (2008), L'argument statistique. Vol. 1 : Pour une sociologie historique de la quantification, Paris, Mines ParisTech.

Dodier N. (1993), L'expertise médicale. Essai de sociologie sur l'exercice du jugement, Paris, Métailié.

Dubas F. (2004), La médecine et la question du sujet. Enjeux éthiques et économiques, Paris, Les Belles Lettres.

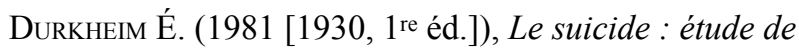
sociologie, Paris, Presses universitaires de France.

Engel G. (1980), " The clinical application of the biopsychosocial model », American Journal of Psychiatry, $\mathrm{n}^{\circ}$ 135, pp. 535-544.

FAURE O. (2005), «Les sages-femmes en France au XIX ${ }^{\mathrm{e}}$ siècle : médiatrices de la nouveauté», in Bourdelais P., Faure O. (dir.), Les nouvelles pratiques de santé, XVIII $-X X^{e}$ siècles, Paris, Belin, pp. 157-174.

Foucault M. (1963), Naissance de la clinique: une archéologie $d u$ regard médical, Paris, Presses universitaires de France.

Freidson E. (1970), La profession médicale, Paris, Payot.

Friedmann G. (1950), Où va le travail humain ?, Paris, Payot.

Gadéa C., Hardy-Dubernet A.-C. (dir.) (2005), «De "faire médecine" à "faire de la médecine"», Document de travail, Série Études, no 53, Drees.

Godefroy P. (2013), «Les affectations des étudiants en médecine à l'issue des épreuves classantes nationales en 2012 », Études et résultats, no 852, Drees.

Hardy A.-C. (2013), Travailler à guérir. Sociologie de l'objet du travail médical, Rennes, Presses de l'École des hautes études en santé publique. 
Hardy-Dubernet A.-C., Faure Y. (2006), «Le choix d'une vie... Étude sociologique des choix des étudiants de médecine à l'issue de l'examen classant national en 2005 », Document de travail, Série Études, no 66, Drees.

Herzlich C., Bungener M., Paicheler G., Roussin P., Zuber M.-C. (1993), Cinquante ans d'exercice de la médecine en France. Carrières et pratiques des médecins français 1930-1980, Paris, éditions Inserm/Doin.

Hughes E. C. (1996), Le regard sociologique: essais choisis, textes rassemblés et présentés par J.-M. Chapoulie, Paris, École des hautes études en sciences sociales.

JACQues B. (2007), Sociologie de l'accouchement, Paris, Presses universitaires de France.

Jamous H. (1969), Sociologie de la décision. La réforme des études médicales et des structures hospitalières, Paris, Éditions du CNRS.

LABARThe G., HÉrault D. (2003), «Les étudiants inscrits en médecine en janvier 2002», Études et résultats, $\mathrm{n}^{\mathrm{0}} 244$, Drees.

LEWIN F. (2004), «L'obstétricien, les parents et le fœtus malade», in Séguret S., Le consentement éclairé en périnatalité et en pédiatrie, Paris, Eres, pp. 79-82.

ONDPS (2008), Rapport annuel 2006-2007 de l'Observatoire national de la démographie des professions de santé, Tome 1: La médecine générale, Paris, Ministère de la Santé, de la Jeunesse, des Sports et de la Vie associative.

Observatoire régional de la santé des Pays de la Loire (ORS), Union RÉGIONALE des mÉdecins LibérauX des Pays DE LA LOIRE (URML) (2010), Vie professionnelle, vécu du travail et état de santé des médecins généralistes des Pays de la Loire, vague d'enquête 4 - 2008 - Rapport détaillé; disponible en ligne à l'adresse : http://www. santepaysdelaloire.com/ors/sites/ors/files/publications/ PanelMG/2010panel1_v4_rapport.pdf; consulté le 14 avril 2015.

Peneff J. (1997), «Le travail du chirurgien : opérations à cœur ouvert», Sociologie du travail, vol. 39, nº 3, pp. 265-296.

Pinell P. (2004), «Champ médical et processus de spécialisation», Actes de la recherche en sciences sociales, no 156-157, pp. 4-36.

Piotet F. (2002), La révolution des métiers, Paris, Presses universitaires de France.

Schlumbohm J. (2002), «Comment l'obstétrique est devenue une science. La maternité de l'université de Göttingen, 1751-1830», Actes de la recherche en sciences sociales, no 143 , pp. 18-30.

SiCARD D. (2013a), «Les médecins au 1er janvier 2013», Document de travail, Série Statistiques, no 179, Drees.

SiCARD D. (2013b), «Les professions de santé au 1 er janvier 2013 », Document de travail, Série Statistiques, no 183 , Drees.

Strauss A. (1992), La trame de la négociation. Sociologie qualitative et interactionniste, textes réunis et présentés par I. Baszanger, publiés entre 1961 et 1985, Paris, L'Harmattan.

Weisz G. (2006), Divide and conquer. A comparative history of medical specialization, New York, Oxford, Oxford University Press.

Weisz G., Löwy L. (2005), « French hormones: progestins and therapeutic variation in France », Social science and medicine, $\mathrm{n}^{\circ} 60$, pp. 2609-2622.

Zolesio E. (2012), Chirurgiens au féminin? Des femmes dans un métier d'hommes, Rennes, Presses universitaires de Rennes. 